\title{
Intravaginal and intrarectal microbicides to prevent HIV infection
}

$\triangle$ bout 40 million people worldwide have HIV infection (UNAIDS 2004 report [www.unaids.org/bangkok2004/ report.html]). In 2003 alone, almost 5 million new infections occurred, more than $90 \%$ of which were sexually acquired. Nearly $50 \%$ of the worlds' $\mathrm{HIV}$-infected people are women, and over $30 \%$ of HIVpositive women in some subSaharan African countries are teenagers. More than 1000 $\mathrm{HIV}$-infected babies are born each day, often to teenaged mothers. Despite the effectiveness and availability of the condom, the HIV epidemic continues to spread. New prevention strategies are urgently needed. Topical microbicides are being developed as a possible new therapeutic approach to prevent HIV infection. They are formulated as gels, foams, films or vaginal rings designed to be inserted into the vagina or rectum and meet the urgent need for an effective female-controlled method of HIV prevention. More than 60 potential microbicides are being assessed in preclinical and clinical trials.

Improved understanding of the HIV life cycle (binding, fusion, reverse transcription, DNA integration, host-cell activation, transcription, translation, protein assembly, budding and viral release), of the mechanisms for HIV infection and transmission across mucosal epithelium (Fig. 1) and of the tropism of different viral strains opens new possibilities for antiviral interventions. This includes recognition of the role coreceptors play in HIV binding and of the particular role dendritic cells play in transmitting the virus to the lymphatic system. In addition to the high affinity the viral envelope glycoprotein gp120 has for binding to the CD4 protein on the host cell, a coreceptor (e.g., the
CCR5 used by macrophagetropic strains or the CXCR4 used by T-cell-tropic and Tcell-line-adapted strains) is needed for HIV to enter the cell. Dendritic cells are immune cells with thread-like tentacles or dendrites that "capture" antigens and transport them to $T$ cells. Examples of dendritic cells include Langerhans cells found in the skin and mucosal membrane, and follicular dendritic cells found in lymphoid tissue. Dendritic cells carry the CD4 surface marker, and the majority of dendritic cells also carry CXCR4, DC-SIGN and CCR5 that effectively bind HIV through gp 120. The bound $\mathrm{HIV}$ migrates to lymphatic areas, where T4 lymphocytes can be productively infected.

Microbicides can act in various ways (Fig. 2). Microbicides

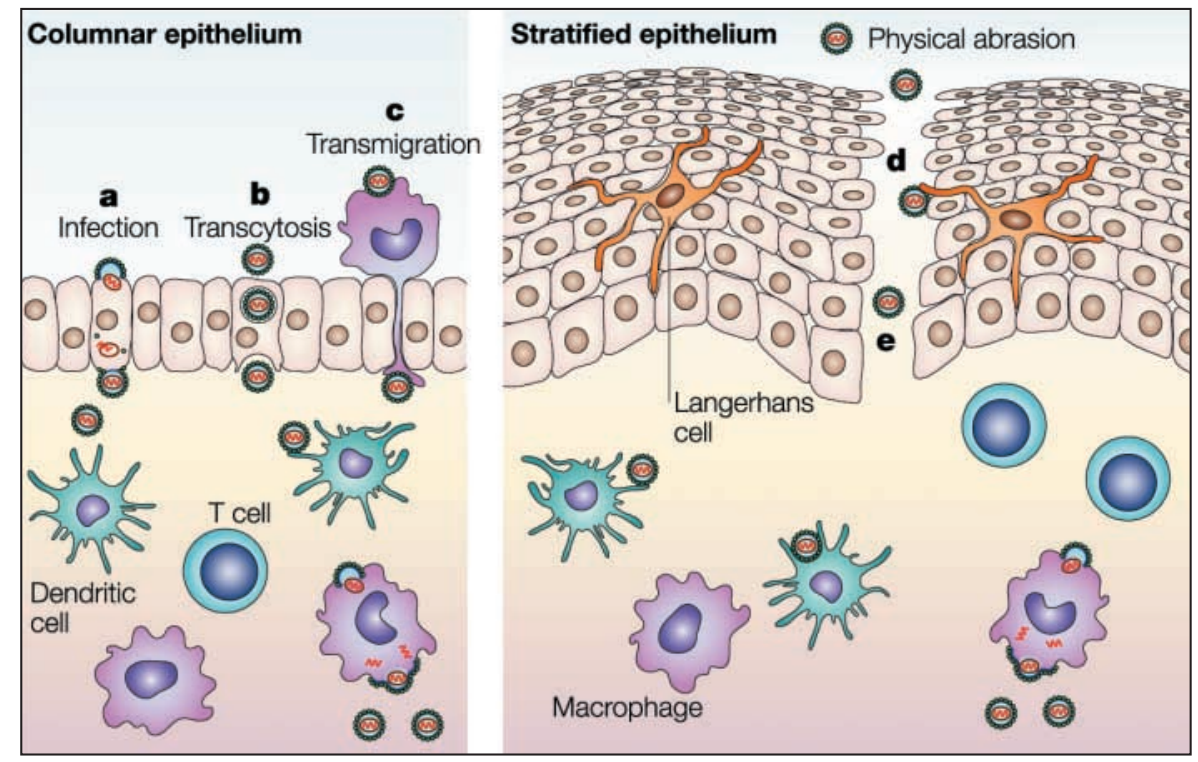

Fig. 1: Potential mechanisms for HIV transmission across mucosal epithelium. (a) Direct infection of epithelial cells; (b) transcytosis through epithelial cells or specialized microfold cells; (c) epithelial transmigration of infected donor cells; (d) uptake by intraepithelial Langerhans cells; (e) circumvention of the epithelial barrier through physical breaches. Successful transfer of the virus across epithelial barriers would result in viral uptake by migratory dendritic cells (by DC-SIGN or other mannose C-type lectin receptors) and subsequent dissemination to T cells in the lymphatic system or localized mucosal infection, leading to recruitment of additional susceptible cells. Reproduced, with permission, from Shattock RJ, Moore JP. Inhibiting sexual transmission of HIV-1 infection. Nature Rev Microbiol 2003;1:25-34. 
membranes of the vaginal and cervical epithelium and cause erosions and lesions, leading to an increased risk of HIV infection. The therapeutic window of nonspecific microbicides appears to be rather limited. Spermicides, including nonoxynol-9, belong in this category. Nonoxynol-9 is not effective in preventing HIV infection. On the contrary, in a randomized controlled clinical trial involving sex workers, it increased the risk of HIV infection. ${ }^{1}$ Currently, a new nonspecific agent, C31G (Savvy), with a higher therapeutic index than nonoxynol-9 is being tested in a phase III HIV prevention trial (Table 1).

The buffering agents (BufferGel and ACIDFORM) also belong to the category of nonspecific products. They act by effecting a local $\mathrm{pH}$ change that results in inactivation of the microorganism. A phase IIb trial of BufferGel is planned (Table 1). ACIDFORM is being assessed for long-term safety and prevention of STDs (excluding HIV infection) in combination with the diaphragm.

\section{More specific microbicides}

A variety of anionic substances that target the adsorption and fusion process of the virus (Box 1) are under investigation. They bind to the viral envelope through their negative charges and block cell entry. They may be effective not only on HIV but also on other enveloped viruses such as herpesviruses. Although rather efficient in preventing contact between the cell-free virus and its target cell, they are generally less efficient in preventing transmission of the cell-associated virus to uninfected cells. Because of the rather limited general toxicity of these anionic substances, their therapeutic window is higher than that of the detergents or surfactants. Several polyanions have shown an anticoagulant effect in vitro, but this has not been shown in women using these products intravaginally. ${ }^{2,3}$ Anionic products are the most

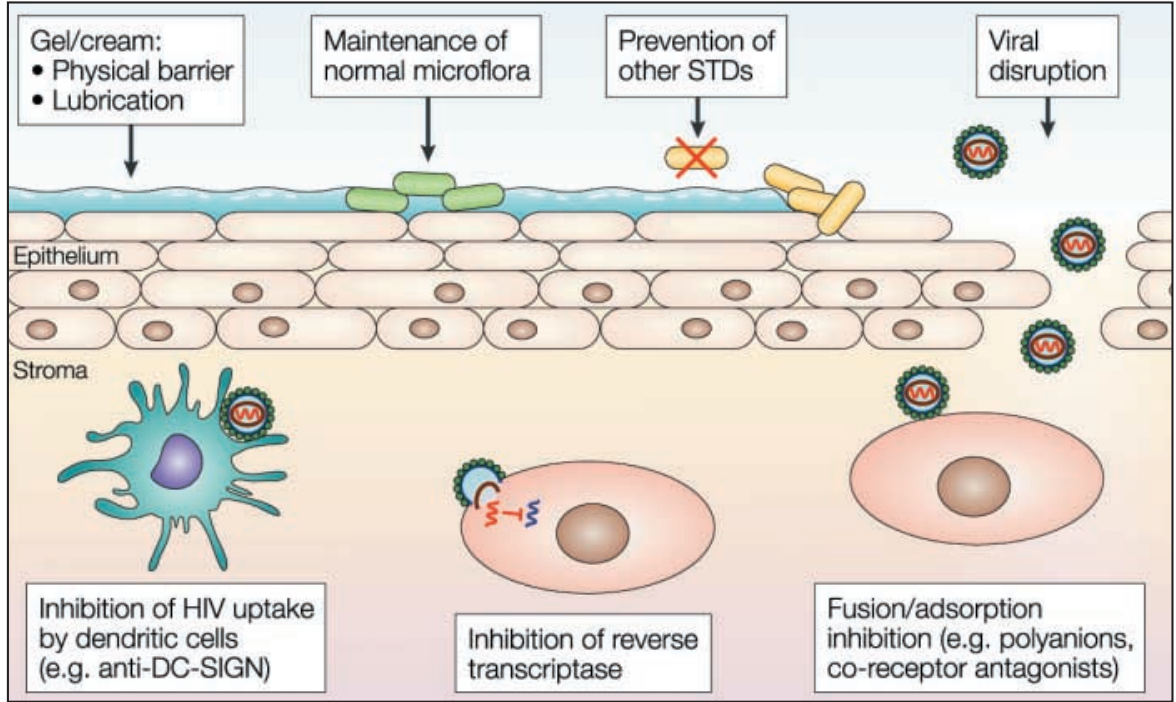

Fig. 2: Potential mechanisms of action for microbicide compounds. Microbicides that act as a lubricant coat the epithelial surface, which might reduce the risk of trauma and provide a physical barrier against viral infection. Prevention (or treatment) of other STDs can reduce the risk of HIV transmission by reducing the risk of epithelial inflammation and ulceration. In the vagina, maintaining the normal flora, and therefore maintaining the vaginal $\mathrm{pH}$ at virucidal levels ( $\mathrm{pH}$ less than 4.5), could also reduce the risk of transmission. Once the virus has crossed the epithelial barrier, potential microbicidal strategies include targeting HIV uptake by dendritic cells, targeting HIV adsorption and fusion, and targeting reverse transcriptase and integration into the host cell genome. Reproduced, with permission, from Shattock RJ, Moore JP. Inhibiting sexual transmission of HIV-1 infection. Nature Rev Microbiol 2003;1:25-34. advanced of the microbicides in clinical development. One product - Carraguard - is already in a phase III HIV prevention trial ('Table 1). This product has no contraceptive effect. Two others (cellulose sulfate and PRO 2000) will soon be tested in phase III trials. A contraceptive phase II trial is underway for cellulose sulfate.

\section{Highly specific microbicides}

HIV-specific microbicides should preferentially block the viral replication cycle at a step before integration of the proviral genome into the target cell (Box 1). Several phases of the viral life cycle (virus entry, reverse transcription of the viral RNA genome, proviral DNA integration in the host-cell chromosomes) can be a target for microbicidal intervention.

Soluble CD4, a highly specific antibody against the CXCR4 and CCR5 (co)receptors, and cytokines that represent the natural ligands of the coreceptors CXCR4 or CCR5 have been shown to efficiently inhibit entry of HIV into its target cells. However, their in vitro activity often depends on the virus source and cell type investigated. Because of their peptidic nature and the presence of proteolytic enzymes in vaginal secretions, they may be less attractive as potential microbicides than small molecules that specifically interact with CXCR4, and particularly with CCR5 (Box 1). Natural polypeptides such as mannose and $N$-acetylglucosamine-specific plant lectins (agglutinins stable at acidic $\mathrm{pH}$ levels of about 4.5 and temperatures of about $50^{\circ} \mathrm{C}$ ) and the cyanobacterial mannose-specific lectin cyanovirin specifically bind to the sugar moieties present on the envelope gp120 of HIV. ${ }^{4}$ These molecules have been shown in cell cultures to efficiently block both infection of cells by free virus particles and virus transmission between virus-infected and uninfected cells. However, the cost of mass production of these proteins may hamper a wide ap- 
plication as microbicide drugs. Therefore, attempts are underway to express these proteins by commensal bacteria of the vaginal flora (i.e., lactobacillus) in order to create a local microbicidal environment upon inoculation.

Interestingly, some modified glycopeptide antibiotics related to vancomycin, teicoplanin and eremomycin have recently been shown to prevent both HIV infection and transmission in cell culture, possibly by interacting with the viral envelope gp120. A heterogeneous variety of other substances (Box 1) have been shown in cell culture to prevent virus infection and transmission at nontoxic concentrations, but in vivo activity still needs to be demonstrated. If specific inhibitors of the gp41-driven viral fusion process other than the expensive peptide T-20 (enfuvirtide) become available, they may represent a powerful tool to efficiently prevent $\mathrm{HIV}$ infection.

Once the virus has entered the intracellular environment, it can be stopped from productive infection only by inhibiting the virus-encoded reverse transcriptase or integrase enzymes. Several known reverse transcriptase inhibitors (Box 1) are currently the subject of clinical phase I and II trials as potential microbicides. The HIV-1 non-nucleoside reverse transcriptase inhibitors (NNRTIs) have the advantage of a high therapeutic window. The presence of a lipophylic tightbinding NNRTI at the site of virus infection or transmission may result in a virtual direct inactivation of the reverse transcriptase in the virus particle. Because of their lipophylic nature, NNRTIs may easily be incorporated into the drugexposed target cell membrane, which creates a local protective barrier if the virus tries to enter the cells.

In contrast to NNRTIs, many of the earlier integrase inhibitors have a limited therapeutic window and may not even reach their presumed antiviral target because of their chemical properties. However, the more recently developed integrase inhibitors (Box 1) may be more effective as microbicides.

\section{Systemic absorption and drug resistance}

Absorption of microbicides used intravaginally or intrarectally may result in systemic side effects or viral drug resistance or both. The development of viral drug resistance should be avoided as much as possible. Whereas no evidence of systemic absorption was found in safety studies of anionic compounds, such evidence was found after intravaginal application of the reverse transcrip- tase inhibitors tenofovir, UC781 and TMC-120. However, it is currently unclear whether such plasma drug levels are associated with a substantial risk of development of drug resistance.

\section{Drug combinations}

Because of the complexity of viral entry and spread in the body, a cocktail of several (at least up to 3) drugs may be needed that will intervene at different steps in the virus infection and entry process to optimally prevent transmission. A combination of several drugs may offer the advantage of using lower concentrations of

Box 1: Types of candidate microbicides being developed for intravaginal and intrarectal use as a possible new therapeutic approach to preventing HIV transmission

\section{Nonspecific microbicides}

Detergents or surfactants

- Nonionic compounds (e.g., nonoxynol-9, chlorhexidine)

- Anionic compounds (e.g., sodium lauryl sulfate, sodium dodecyl sulfate, monocaprin)

- Cationic compounds (e.g., benzalkonium chloride, C31G, GEDA Plus)

Buffering agents

- BufferGel

- ACIDFORM

More specific microbicides

Anionic polymers*

- Sulfated polysaccharides (e.g., dextrin sulfate, heparin, cellulose acetate phtalate, cellulose sulfate, carrageenan)

- Sulfonated polymers (e.g., PRO-2000, PAVAS, PVAS)

- Polycarboxylates

Othert

Highly specific anti-HIV microbicides

Entry inhibitors

- Soluble CD4 (e.g., soluble CD4-lgG)

- CXCR4 antagonists (e.g., SFD-1, bicyclams)

- CCR5 antagonists (e.g., RANTES derivatives, LD78 $\beta$ isoform of MIP- $1 \alpha, \mathrm{SCH}-\mathrm{C}$, TAK779)

- Glycoprotein 120 (gp120)-recognizing agents (e.g., plant lectins and cyanovirin from Cyanobacterium; modified glycopeptide antibiotics related to vancomycin, teicoplanin and eremomycin; neutralizing antibodies)

- gp41-interacting agents (i.e., T-20 [enfuvirtide])

Reverse transcriptase inhibitors

- NtRTIs (e.g., [R]-PMPA [tenofovir])

- NNRTIs (e.g., UC-781, TMC-120 [dapivirine])

Integrase inhibitors

- Variety of compounds (i.e., S-1360; C-731,988)

Note: SFD-1 $=$ stromal cell-derived factor 1, MIP- $1 \alpha=$ macrophage inflammatory protein $-1 \alpha$, NtRTI = nucleotide reverse transcriptase inhibitor, NNRTI = nonnucleoside reverse transcriptase inhibitor *Anionic polymers act as inhibitors of HIV adsorption or fusion, or both, but are less specific than the substances listed under "highly specific anti-HIV microbicides" in that they may also interact with pathogens other than HIV.

tIncludes defensins, protegrin, hypericin, betulinic acid and plant extracts. 
Table 1: Clinical trials of microbicide formulations currently underway

\begin{tabular}{llll}
\hline Product & \multicolumn{1}{c}{ Primary research group } & \multicolumn{1}{c}{ Trial countries } & \multicolumn{1}{c}{ Study design } \\
\hline $\begin{array}{l}\text { BufferGel and } \\
\text { PRO 2000 0.5\% }\end{array}$ & HIV Prevention Trial Networks & $\begin{array}{l}\text { India, Malawi, South Africa, } \\
\text { Tanzania, United States, Zambia, } \\
\text { Zimbabwe }\end{array}$ & 4-arm phase II/IIb \\
\hline Cellulose sulfate & $\begin{array}{l}\text { Global Microbicide Project } \\
\text { (GMP-CONRAD)* } \\
\text { Family Health International } \\
\text { and GMP-CONRAD }\end{array}$ & $\begin{array}{l}\text { Benin, Burkina Faso, India, } \\
\text { Kenya, South Africa, Uganda } \\
\text { Nigeria }\end{array}$ & 2-arm phase III \\
\hline Carraguard & $\begin{array}{l}\text { Population Council } \\
\text { Pro 2000 0.5\% and 2\% }\end{array}$ & $\begin{array}{l}\text { SK Microbicides Development Africa } \\
\text { Programme* }\end{array}$ & $\begin{array}{l}\text { Cameroon, South Africa, } \\
\text { Tanzania, Uganda, Zambia }\end{array}$ \\
\hline Savvy (C31G) & Family Health International & Nigeria, Ghana & 2-arm phase \\
\hline
\end{tabular}

*Planned to start in 2005.

each drug, which would offer a reasonable antiviral efficacy but a lower risk of toxic effects. Still, design challenges abound. Not only are the complementary mechanisms of action of the drugs important considerations, but so is the compatibility of different drugs in a single microbicidal formulation.

\section{Conclusion}

There is a compelling need to develop and evaluate novel microbicides for intravaginal and intrarectal use that are directed against different targets and steps in HIV infection and transmission. A single microbicidal agent will likely not be sufficient, and instead a combination of several drugs will probably be needed to ensure effective protection. Interestingly, given the tremendous burden of HIV infection on the population, mathematical models have shown that even use of a microbicide that is only moderately effective may have a pronounced impact on public health. Issues of safety and effectiveness aside, additional challenges posed by the prospect of microbicides include avoiding the possibility of decreased condom use, creating a willing market, educating women and men, and providing the product at an affordable price.

\section{Jan Balzarini}

Rega Institute for Medical Research Leuven, Belgium

Lut Van Damme

CONRAD

Arlington, Vt.

\section{References}

1. Van Damme L, Ramjee G, Alary M, Vuylsteke B, Chandeying V, Rees H, et al; COL-1492 Study Group. Effectiveness of COL-1492, a nonoxynol-9 vaginal gel, on HIV-1 transmission in female sex workers: a randomised controlled trial [published erratum appears in Lancet 2002;360:1892]. Lancet 2002;360(9338):971-7.

2. Van Damme L, Wright A, Depraetere K, Rosenstein I, McKinlay M, Poulter L, et al. A phase I study of a novel potential intravaginal microbicide, PRO 2000 , in healthy sexually inactive women. Sex Transm Infect 2000;76: 126-30.

3. Mauck C, Weiner DH, Ballagh S, Creinin M, Archer DF, Schwartz J, et al. Single and multiple exposure tolerance study of cellulose sulfate gel: a phase I safety and colposcopy study. Contraception 2001;64:383-91.

4. Balzarini J, Hatse S, Vermeire K, Princen K, De Clercq E, Egberink H, et al. Mannose-specific plant lectins from the Amaryllidaceae family qualify as efficient microbicides to prevent infection and transmission of the human immunodeficiency virus. Antimicrob Agents Chemother 2004;48:3858-70. 